\title{
The effects of maribavir on the autophosphorylation of ganciclovir resistant mutants of the cytomegalovirus UL97 protein
}

\author{
Claire D Shannon-Lowe, Vincent C Emery
}

\begin{abstract}
Background: The UL97 protein kinase of human cytomegalovirus phosphorylates the antiviral drug ganciclovir and is the target of maribavir action. A detailed enzyme kinetic analysis of maribavir on the various enzymatic functions of wild type and ganciclovir resistant forms of UL97 is required.

Methods: Wild type and site directed mutant forms of the human cytomegalovirus UL97 gene product were expressed using recombinant baculoviruses and the purified products used to assess the effects of maribavir on the ganciclovir (GCV) kinase and protein kinase (PK) activities.

Results: Maribavir was a potent inhibitor of the autophosporylation of the wild type and all the major GCV resistant UL97 mutants analysed (M460I, H520Q, A594V and L595F) with a mean IC 50 of $35 \mathrm{nM}$. The M460I mutation resulted in hypersensitivity to maribavir with an $\mathrm{IC}_{50}$ of $4.8 \mathrm{nM}$. A maribavir resistant mutant of UL97 (L397R) was functionally compromised as both a GCV kinase and a protein kinase ( 10\% of wild type levels). Enzyme kinetic experiments demonstrated that maribavir was a competitive inhibitor of ATP with a Ki of $10 \mathrm{nM}$.

Discussion: Maribavir is a potent competitive inhibitor of the UL97 protein kinase function and shows increased activity against the M460l GCV-resistant mutant which may impact on the management of GCV drug resistance in patients.
\end{abstract}

\section{Background}

The UL97 gene of human cytomegalovirus encodes a 690 amino acid nuclear serine/threonine protein kinase that is present in the virion and is critical for efficient viral replication [1-8]. The precise function of UL97 in the replication cycle has not been fully elucidated but UL97 has been implicated in replication, viral DNA packaging, nuclear egress, virion morphogenesis and cell cycle manipulation [9-15]. The full range of targets for UL97 kinase action are also undefined although the viral UL44 protein is one viral substrate [16] and pUL97 complexes with the tegument phosphoprotein pp65 (ppUL83) [17]. UL97 also phosphorylates the antiviral nucleosides ganciclovir (GCV) and acyclovir [18-21], the former being the major drug currently used to control HCMV replication in vivo [22,23]. In the era preceding the deployment of

\footnotetext{
* Correspondence: v.emery@medsch.ucl.ac.uk

Department of Infection, Centre for Virology, UCL (Royal Free Campus Campus), Rowland Hill Street, Hampstead, London NW3 2QG, UK
}

highly active antiretroviral therapy for HIV infection, long term GCV therapy for cytomegalovirus retinitis led to the development of GCV resistance in up to $40 \%$ of patients [24] although the frequency appears to be lower in transplant recipients receiving GCV prophylaxis [24-27]. A number of studies have investigated the molecular basis for resistance of HCMV to GCV and show that a small number of mutations in UL97 (at amino acids M460, H520, A594 and L595) account for the majority $(\sim 85 \%)$ of clinically significant drug resistance [28-31]. Subsequent to the development of UL97 mutations, resistance at the DNA polymerase locus can also occur [32-34]. In vivo dynamic modelling has shown that these mutant viruses are less fit than their wild type counterparts and, in the absence of selective drug pressure, wild type virus is between $5-10 \%$ more fit than the GCV resistant mutants [35].

The benzimidazole class of compounds are novel inhibitors of HCMV replication. The progenitor, BDCRB (2bromo-5,6-dichloro-1-( $\beta$-D-ribofuranosyl benzimidazole)

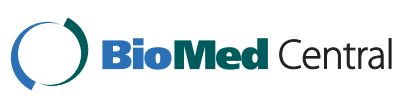

(c) 2010 Shannon-Lowe and Emery; licensee BioMed Central Ltd. This is an Open Access article distributed under the terms of the Creative Commons Attribution License (http://creativecommons.org/licenses/by/2.0), which permits unrestricted use, distribution, and reproduction in any medium, provided the original work is properly cited. 
inhibits the packaging of HCMV genomes via the UL89 and the UL56 terminase proteins [36] whereas the related compound maribavir (5,6-dichloro-2-(isopropylamino)-1beta-L-ribofuranosyl-1H-benzimidazole; also known as 1263W94 and benzamidavir) targets the protein kinase activity of UL97 (37). Maribavir has potent antiviral activity against HCMV [37] and EBV [38], with an $\mathrm{IC}_{50}$ approximately 4-to 10-fold lower than that observed for GCV against HCMV replication in vitro and is active against HCMV strains resistant to ganciclovir, foscarnet and cidofovir [39]. Cell culture of HCMV in the presence of maribavir routinely leads to drug resistance involving a single amino acid change in UL97: L397R [37]. However, recently, using a clinical isolate with a mutation in the exonuclease domain of the viral DNA polymerase additional UL97 mutations at amino acids 411 ( $\mathrm{H}$ to $\mathrm{L} / \mathrm{Y} / \mathrm{N}$ ), 409 ( $\mathrm{T}$ to $\mathrm{M}$ ) and 353 (V to $\mathrm{A}$ ) have also been described $[40,41]$. These mutations cluster more closely to the proposed ATP binding domain of UL97. Maribavir has undergone phase I and II clinical trials and phase III prophylaxis trials in solid organ and stem cell transplant recipients [42-44]. Mutations which contribute to drug resistance against maribavir have also been identified in UL27 although the precise function of this protein in the viral life cycle remains to be fully defined [45].

At present, a detailed enzyme kinetic analysis of drug resistant UL97 mutants has not been undertaken. In the present study, we have used recombinant baculovirus systems to produce UL97 protein of high purity to perform a detailed biochemical analysis of the kinetics of autophosphorylation of a series of the clinically relevant UL97 GCV resistant mutants and a selection mutations in conserved protein kinase domains in UL97 to determine the effects of maribavir on the autophosphorylation capacity of these mutants. The data provide insight into the mechanism of action of maribavir and highlight the impact that certain GCV resistant mutations have on the inhibitory profile of maribavir.

\section{Methods}

\section{Cells and viruses}

Spodoptera frugiperda 21 (Sf21) cells (Invitrogen) were maintained in TC100 medium (Life Technologies, Paisley Scotland) supplemented with $10 \%$ fetal bovine serum, $50 \mathrm{IU}$ of penicillin per $\mathrm{ml}$, and $50 \mu \mathrm{g}$ of streptomycin per ml. Wild-type linearized Autographa californica multiple nuclear polyhedrosis virus (AcMNPV; Invitrogen, Scotland) was used to construct the recombinant baculoviruses. The Towne strain of cytomegalovirus was propagated in primary human embryonic lung fibroblasts maintained in DMEM medium (Life Technologies, Scotland) supplemented with $10 \%$ fetal calf serum, $2 \mathrm{mM}$ L-glutamine, $100 \mathrm{IU}$ of penicillin per $\mathrm{ml}$, and $100 \mu \mathrm{g}$ of streptomycin per $\mathrm{ml}$ in $5 \% \mathrm{CO} 2$.

\section{Cloning of the HCMV UL97 ORF}

Human embryonic lung fibroblasts were infected with the Towne strain of HCMV at a multiplicity of infection (m.o.i.) of 5 and $72 \mathrm{~h}$ later DNA extracted using the Wizard genomic purification kit (Promega, Southampton UK). The extracted DNA (1 ug) was used as the template for a PCR reaction to generate the full length UL97. The primers (5'-GGG GTA CCC ATG TCC TCC GCA CTT CGG TCT CGG and 5'-CCC AAG CTT TTA CTC GGG GAA CAG TTG GCG GCA) were designed to generate an amplicon with a 5' Kpn I and a 3' Hind III restriction site. PCR conditions consisted of $95^{\circ} \mathrm{C}$ for $5 \mathrm{~min}$ followed by cycling at $60^{\circ} \mathrm{C}$ for $3 \mathrm{~min}$, $72^{\circ} \mathrm{C}$ for 10 minutes and $95^{\circ} \mathrm{C}$ for 3 minutes using the Bioline (UK) high fidelity Taq polymerase mixture (Bio$\mathrm{X}$-Act) according to the manufacturer's instructions. The 2160 bp DNA amplicon was purified using the Wizard $^{\mathrm{rm}}$ PCR prep DNA purification system (Promega, UK) and ligated into the pGEM-T Easy vectors (Promega, UK) for subsequent sequencing of the entire UL97 ORF. The pGEM-UL97 construct was restricted with Kpn I and Hind III, the UL97 fragment purified and ligated into the pMelBac $\mathrm{C}$ or pBluBacHis $\mathrm{C}$ transfer vectors (Invitrogen, Scotland), which had been similarly restricted enabling directional cloning of the UL97 in the correct open reading frame. The pMelBac transfer vector allowed the incorporation of a $\mathrm{N}$-terminal honey bee melittin secretion signal onto the UL97 protein while the pBluBacHis incorporated an N-terminal hexahistidine tag.

\section{Site directed mutagenesis of the UL97 ORF}

Point mutations were introduced into the pMelUL97 transfer vectors using the GeneEditor ${ }^{\text {TM }}$ in vitro Site Directed Mutagenesis system (Promega, Southampton, UK). Briefly, $100 \mathrm{ng}$ of denatured template DNA was added to an annealing solution of $0.25 \mathrm{pmol}$ of the appropriate phosphorylated selection oligonucleotide (top strand), 1.25 pmol of phosphorylated mutagenic oligonucleotide and $10 \times$ TM buffer $(100 \mathrm{mM}$ Tris, 100 $\mathrm{mM} \mathrm{MgCl}_{2}$ ). Annealing was performed for $15 \mathrm{~min}$ at $37^{\circ} \mathrm{C}$ and mutant strand synthesis and ligation performed at $37^{\circ} \mathrm{C}$ for $90 \mathrm{~min}$ following addition of $10 \times$ synthesis buffer $(100 \mathrm{mM}$ Tris- $\mathrm{HCl}, \mathrm{pH} 7.5,5 \mathrm{mM}$ dNTP, 10 mM ATP, 20 mM DTT), T4 DNA polymerase, and T4 DNA ligase according to manufacturers instructions. Following the mutagenesis reaction, $1.5 \mu \mathrm{l}$ of each reaction was transformed into $\mathrm{BMH}$ 71-18 mutS E.coli, the plasmid harvested and transformed into JM109 E.coli in an appropriate antibiotic selection mix to ensure good segregation of the mutant and wild type plasmids. The mutant UL97 ORFs were DNA sequenced to confirm the specific introduction of the mutation and the absence of other mutations and recombinant 
baculoviruses generated as described below. The primers used to introduce the desired mutations were as follows: K355Q (5'-CGC GTG GTC GAG GTG GCG CG); L397R (5'-CGC GGT CTG CGC ACG GCC AC); D456A (5'-TGC CAC TTT GCC ATT ACA CCC); M460I (5'-C ATT ACA CCC ATT AAC GTG C); N461G (5'-ACA CCC ATG AAA GTG CTC ATC); H520Q (5'-GAA TGT TAC CAG CCT GCT TTC C); A594T (5'-C TGC CGC ACG TTG GAG AAC GG); L595F (5'-C TGC CGC GCG TTT GAG AAC GG); H662L(5'-ACC ATG CTG CTC GAA TAC GTC) and V665I (5'-CAC GAA TAC ATC AGA AAG AAC G).

Production of recombinant baculoviruses expressing wild type and mutant forms of UL97

Recombinant baculoviruses were constructed by cotransfection of the transfer vectors with linear, wild type AcMNPV DNA into Sf21 cells using InsectinPlus ${ }^{\mathrm{Tx}}$ liposomes (Invitrogen, Scotland). Recombinant baculoviruses BVBMUL97 or BVBBHUL97 were then harvested 72 hours post transfection and expanded by two rounds of plaque purification to high titre stocks. Insertion of the UL97 and absence of the wild type AcMNPV were confirmed by PCR using primers either side of the cloning sites. Expression of the wild type or mutant UL97 proteins was assessed using SDS-PAGE and Western blotting following infection of Sf21 insect cells at an m.o.i. of 10. After $72 \mathrm{~h}$, cells were lysed and proteins were separated by SDS-PAGE and electrophoretically transferred onto a polyvinylidene difluoride (PVDF; Bio-Rad) membrane by using a semi-dry blotter (Pharmacia Biotech, Buckinghamshire, UK). The membrane was incubated for $1 \mathrm{~h}$ at room temperature in blocking buffer (3\% bovine serum albumin (Sigma Chemicals, Dorset, UK) in TBS [10 mM Tris- $\mathrm{HCl}, \mathrm{pH} 7.5$, $150 \mathrm{mM} \mathrm{NaCl}]$ ), then washed with $0.05 \%$ Tween 20 $0.1 \%$ Triton X-100 in TBS. The membrane was incubated with a 1/500 dilution of mouse UL97-specific polyclonal antisera (a gift from Dr D Michel, Universitatsklinikum Ulm, Abteilung Virologie, Ulm, Germany) in blocking buffer for $1 \mathrm{~h}$ at room temperature then incubated with 1:6,000 dilution of alkaline phosphataseconjugated goat anti-mouse IgG (Bio-Rad, Hemel Hempstead, UK) for $1 \mathrm{~h}$ at room temperature. The membrane was further washed, and immunoreactive bands were visualized by incubation of the membrane in a staining solution consisting of one tablet of 5-bromo4-chloro-3-indolylphosphate-nitroblue tetrazolium chloride (Sigma Chemicals, Dorset, UK) dissolved in $10 \mathrm{ml}$ of distilled $\mathrm{H}_{2} \mathrm{O}$.

Purification of the wild type and mutant UL97 proteins Sf21 insect cells were infected with wild type or mutant UL97 recombinant baculoviruses at an m.o.i. of 10 , incubated at $28^{\circ} \mathrm{C}$ and harvested 72 hours post infection. Cells were sonicated three times in ice cold sonication buffer (50 mM Tris- $\mathrm{HCl}, \mathrm{pH}$ 7.6, $100 \mathrm{mM} \mathrm{NaCl}, 0.1 \%$ Nonidet P-40, 10\% glycerol, $10 \mu \mathrm{g} / \mathrm{ml}$ Aprotinin and Leupeptin and $1 \mathrm{mM}$ Pefabloc), for 10 seconds at 5 microns. Disrupted cells were centrifuged for $10 \mathrm{~min}$ utes at $4^{\circ} \mathrm{C}$ at $13000 \times \mathrm{g}$. Ammonium sulphate was added to the sonicated crude lysate to give a\% saturation of $20 \%-40 \%$, at which the maximum concentration of UL97 was precipitated. The protein precipitate was centrifuged at $3000 \times \mathrm{g}$ for 40 minutes at $4^{\circ} \mathrm{C}$, resuspended in $20 \mathrm{mM}$ Tris- $\mathrm{HCl}, \mathrm{pH} 8.5,100 \mathrm{mM} \mathrm{NaCl}, 0.5 \%$ Sarkosyl and dialysed overnight to give a total of 1/10 000 dilution of the ammonium sulphate. The insoluble proteins were removed by centrifugation and the UL97 dialysed into $20 \mathrm{mM}$ Tris- $\mathrm{HCl}, \mathrm{pH} 8.0,20 \mathrm{mM} \mathrm{NaCl}$ overnight to perform anion exchange chromatography. The soluble protein fraction was applied to a DEAE sepharose column at $4^{\circ} \mathrm{C}$ for 1 hour and the bound protein eluted from the sepharose by buffer containing $0.5 \mathrm{M} \mathrm{NaCl}$. The eluted proteins were concentrated using a Centricon-50 concentration tube. Finally, the UL97 was subjected to immuno-affinity purification.

\section{Phosphorylation of Ganciclovir (GCV) by UL97}

Sf21 insect cells were infected with wild type or mutant UL97 recombinant baculoviruses at an m.o.i. of 10 . After $48 \mathrm{~h}$ incubation at $28^{\circ} \mathrm{C}$, the culture medium was replaced with fresh medium containing $1.0 \mathrm{mM} \mathrm{GCV}$ and $\left[{ }^{3} \mathrm{H}\right]$ GCV $(0.55 \mathrm{Mbq}$, Moravek Radiochemicals, California, USA) After a further 24 hours of incubation, nucleotides were harvested by acid hydrolysis $(0.5 \mathrm{M}$ perchloric acid), neutralisation to $\mathrm{pH} 7.5$ (2.5 $\mathrm{M} \mathrm{KOH}$, $\left.1.5 \mathrm{M} \mathrm{KH}_{2} \mathrm{PO}_{4}\right)$ and centrifugation $(13000 \times \mathrm{g}) .50 \mu \mathrm{l}$ aliquots of supernatant were spotted onto $20 \mathrm{~mm}$ diameter DEAE-coated DE81 chromatographic paper discs and the monophosphorylated GCV allowed to bind. The discs were washed twice in $3 \mathrm{ml} 10 \mathrm{mM}$ ammonium acetate for 10 minutes and twice in $95 \%$ ethanol for 10 minutes. After drying $5 \mathrm{ml}$ of sigmafluor was added (Sigma Chemicals, Dorset, UK) and the radioactivity determined by liquid scintillation counting.

\section{Autophosphorylation of UL97}

Analysis of the autophosphorylation of wild type and mutant UL97 protein species was performed by a standard protein kinase assay as previously described (46). Briefly, purified protein was added to $2 \times$ protein kinase buffer (100 mM Tris $\mathrm{HCl}$, pH9.0, $20 \mathrm{mM} \mathrm{MgCl} 2,10 \mu \mathrm{M}$ ATP, $4 \mathrm{mM}$ DTT, $2 \mathrm{M} \mathrm{NaCl})$ and $370 \mathrm{KBq}\left[\gamma^{32} \mathrm{P}\right]$ ATP (Amersham), made up to $20 \mu \mathrm{l}$ with SDW and incubated at $37^{\circ} \mathrm{C}$. The reactions were terminated by the addition of $2 \times$ SDS sample buffer followed by boiling for 3 minutes. The amount of radiolabelled phosphate 
incorporated by autophosphorylation was analysed by SDS-PAGE and measured using the Biorad Mulitanalyst. The protein kinase assays were initially performed for 2 to 60 minutes to determine the maximum rate of phosphate incorporation, all assays were then performed at the maximum rate which equated to 5 minutes.

\section{Enzyme kinetic analysis}

Enzyme kinetic analysis was performed on the purified wild type and mutant UL97 protein species as above using increasing concentrations of ATP $(2 \mu \mathrm{M}$ to $20 \mu \mathrm{M})$. The amount of incorporated radiolabelled phosphate was plotted against the concentration of ATP in a Lineweaver Burke plot to determine the Km for ATP for each UL97 species. The effect of maribavir upon the rate of radiolabelled phosphate incorporation by wild type or mutant UL97 was determined by protein kinase assays at a fixed concentration of maribavir $(0.5 \mu \mathrm{M})$ as above, or with increasing concentrations of maribavir $(0.01 \mu \mathrm{M}$ to 5.0 $\mu \mathrm{M})$ to determine the $\mathrm{IC}_{50}$ of maribavir for each UL97 species. In order to determine the nature of the inhibition mediated by maribavir, plots of $1 / \mathrm{v}$ vs 1 /ATP with increasing concentrations of maribavir were constructed. Competitive inhibition was evident if the family of lines cconverged on the $y$-axis at $1 / \mathrm{Vmax}$. The change in slope caused by the addition of maribavir was used to calculate the Ki using standard enzyme kinetic equations viz.

$$
K m^{+I}=K m^{-I}\left(1+\frac{[I]}{[K i]}\right)
$$

where $K m^{+I}$ is the apparent $K m$ in the presence of the inhibitor, $\mathrm{Km}^{-I}$ is the $K m$ in the absence of the inhibitor, $K i$ is the dissociation constant of the inhibitor and $[I]$ the concentration of the inhibitor.

\section{Results}

\section{Expression and purification of UL97}

Expression of large amounts of wild type and mutant forms of UL97 for the subsequent biochemical analyses was achieved by using two different recombinant baculovirus expression system using either a C-terminal His tag or fusion of the N-terminus with the honey-bee mellitin signal sequence. All mutant constructs were DNA sequenced to ensure that only the desired mutation had been introduced. Expression levels of UL97 were substantially higher in the honey-bee melittin system rather than the his-tag system and so the former system was used for the subsequent studies (Figure 1). Wild type or mutant UL97 proteins were purified using a combination of ammonium sulphate precipitation followed by anion exchange and affinity chromatography. The various UL97 proteins were purified to > 90\% homogeneity, were expressed at similar levels and were used at the

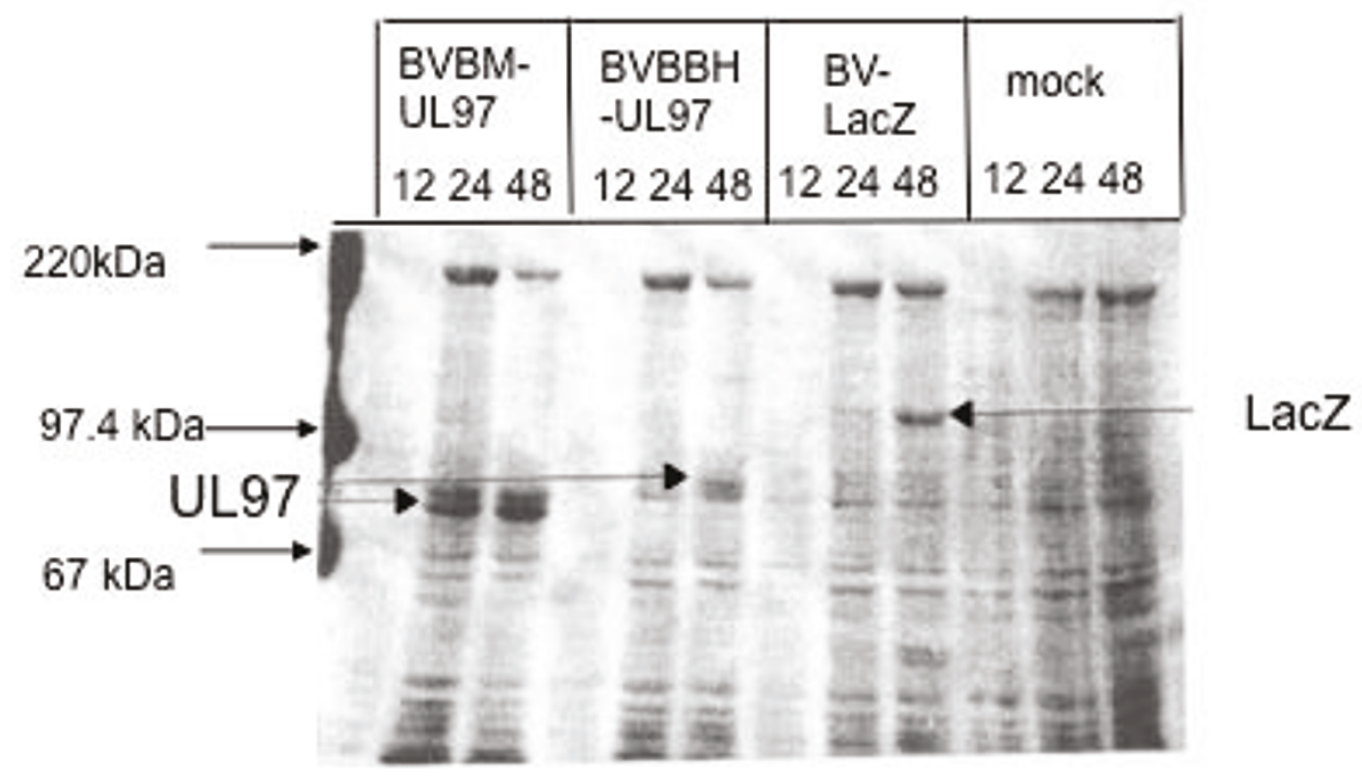

Figure 1 Coomassie Blue stained SDS-PAGE gel showing the time course of expression of the wild type UL97 protein using either the honey-bee mellitin (BVBM-UL97; lanes 1-3) or the BlueBacHis (BVBBH-UL97; lanes 4-6) recombinant baculovirus system illustrating the higher expression levels observed in the honey-bee mellitin system. For comparison, a recombinant baculovirus expressing the LacZ gene (BV-LacZ; lanes 7-10) and mock infected insect cells (mock; lanes 10-12) are also shown. Arrows indicate the UL97 and LacZ proteins and molecular weight markers. 
same protein concentrations for the enzymatic analysis. (Figure 2A and 2B).

\section{GCV phosphorylation by mutant UL97 proteins}

Insect cells were infected with the wild type or mutant UL97-expressing baculoviruses at high multiplicity and after 48 hours, the medium was supplemented with tritiated GCV. The phosphorylation of GCV catalysed by each of the mutant UL97 proteins was compared to wild type GCV phosphorylation (normalised to 100\%). The data summarised in Figure 3 show that GCV phosphorylation by the genotypic GCV-resistant UL97 mutants (M460I, H520Q, A594T and L595F) was reduced to between $10 \%$ and $20 \%$ of the levels of GCV phosphorylation catalysed by the wild type UL97 protein. As expected, mutation at the invariant lysine (K355Q) produced a protein that was unable to phosphorylate GCV.
Although mutation of M460 showed a substantial loss of GCV phosphorylation, mutation of the nearby 456 codon had no effect on GCV phosphorylation. Mutations around the His-X-aromatic-hydrophobic motif at codons H662L and V665I showed a reduction in GCV phosphorylation by $12 \%$ and $72 \%$ of wild type levels respectively. Interestingly, the maribavir-resistant UL97 mutant (L397R) exhibited a significant impairment in GCV phosphorylation (approximately 10\% of wild type).

\section{Kinetic analysis of UL97 autophosphorylation}

Enzyme kinetic analyses were performed on the wild type and mutant UL97 proteins to calculate the Km for ATP. The purified UL97 proteins were incubated in a protein kinase assay for 5 minutes in constant reaction conditions, but with increasing ATP concentrations $(2 \mu \mathrm{M}$ to $20 \mu \mathrm{M})$. The amount of phosphate incorporated was
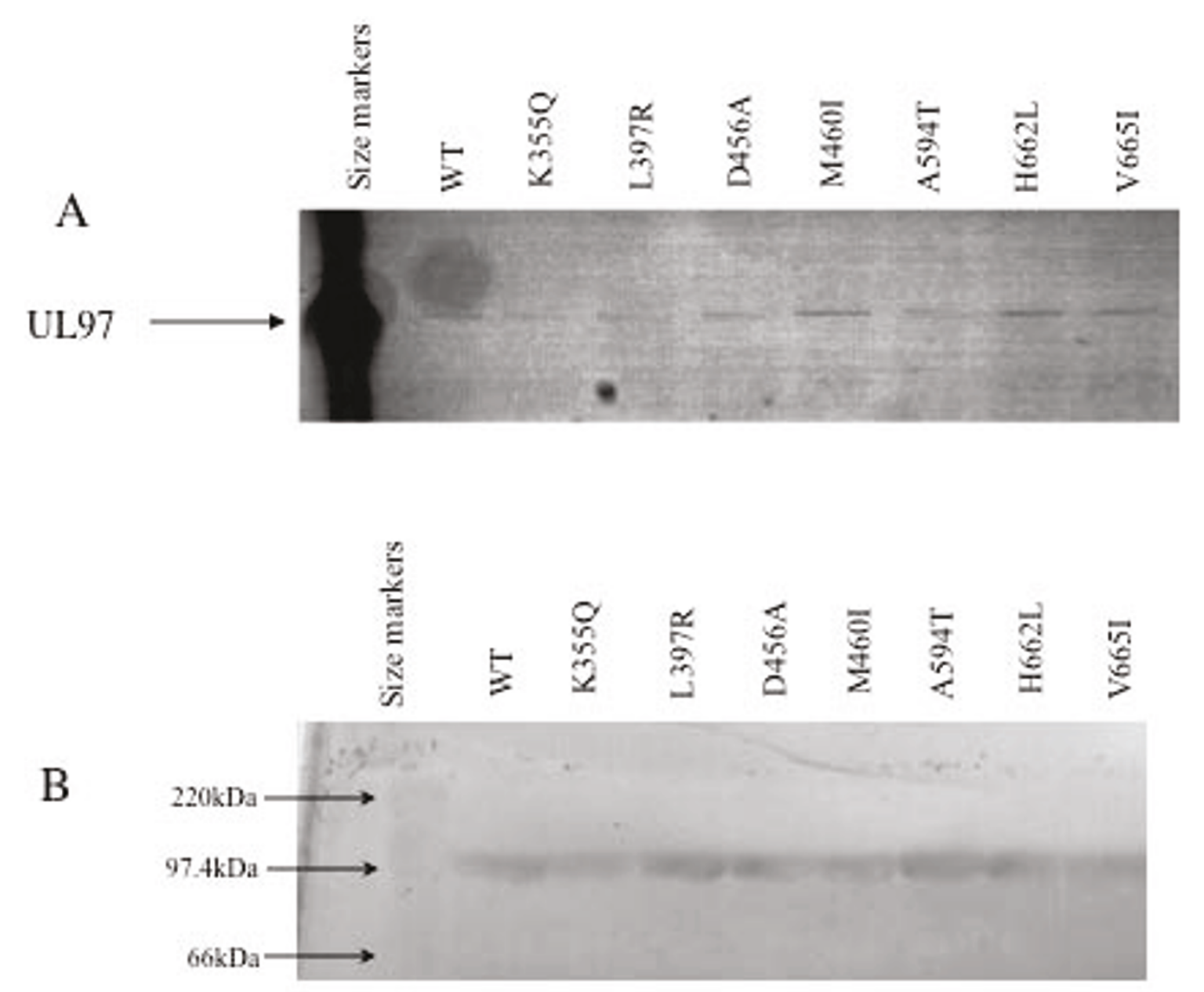

Figure 2 A) SDS-PAGE analysis of purified wild type and a selection of mutant UL97 proteins used in the study. B) Western blot analysis showing the comparable expression levels of wild type and mutant UL97 proteins. 


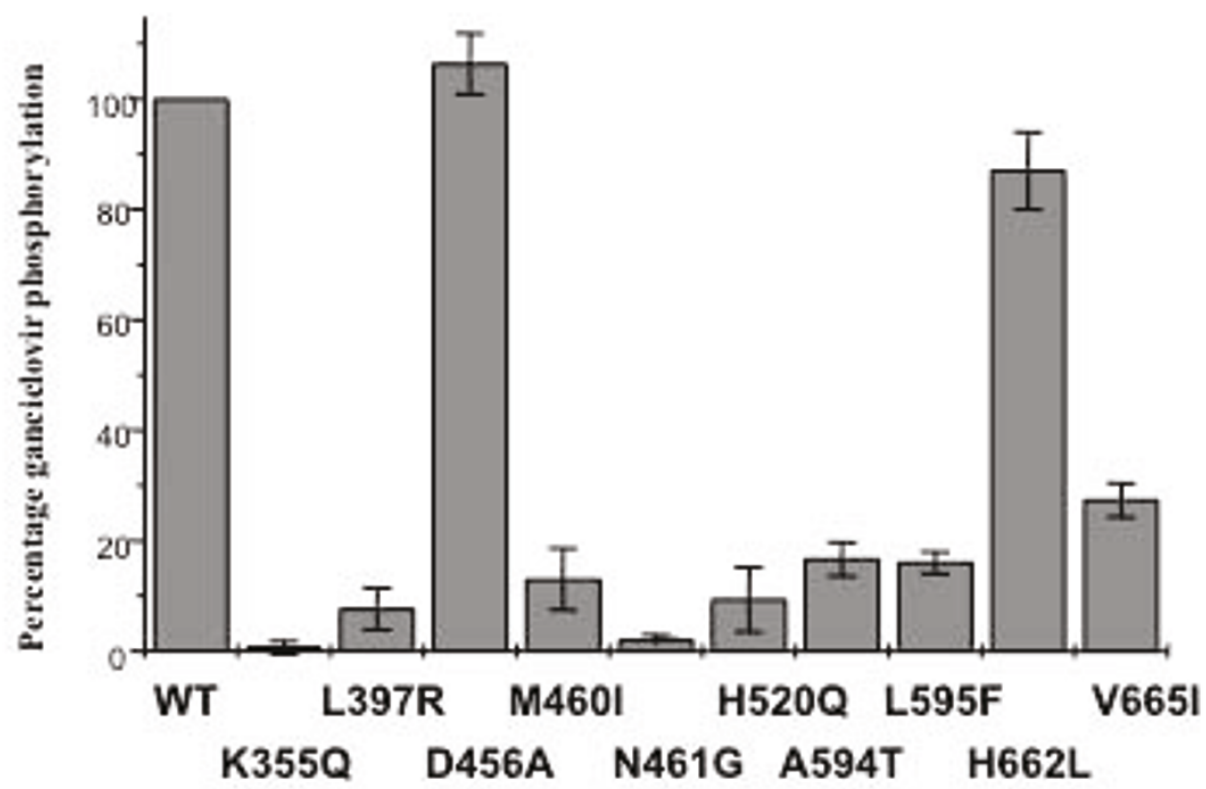

\section{UL97 protein species}

Figure 3 Ganciclovir (GCV) phosphorylation catalysed by the wild type and UL97 mutant proteins (designated by the amino acid change present). Insect cells were infected with the wild type or mutant UL97 expressing baculoviruses at an MOI 10. The medium was supplemented with tritiated GCV at 48 hours. The nucleotides were harvested at 72 hours and bound to DE81 filter paper. The phosphorylated GCV is plotted as percentage phosphorylation compared to the wild type UL97 (set at 100\%). Data are the mean +/- one standard deviation of three experiments.

determined (shown for wild type UL97 in Figure 4) and Lineweaver Burke plots used to calculate the $\mathrm{Km}$ for ATP. In the case of the wild type UL97, the Km for ATP was $27 \pm 2.2 \mu \mathrm{M}$ and for the GCV resistant mutants, the $\mathrm{Km}$ values were as follows: M460I, $28 \pm 2 \mu \mathrm{M}$; H520Q. $25 \pm 2.4 \mu \mathrm{M}$; A594T, $40 \pm 4.2 \mu \mathrm{M}$; L595F, $31 \pm 4.5 \mu \mathrm{M}$. An alternative analysis which gives the relative efficiency of usage of ATP was performed where $\mathrm{Vmax} / \mathrm{Km}$ was calculated. These data showed that the GCV resistant mutant proteins were of similar efficiency as the wild type at utilising ATP than the wild type protein except for the M460I mutant which was substantially less efficient $\left(\mathrm{Vmax} / \mathrm{Km}\right.$ for wild type $=7.2 \mathrm{~s}^{-1} ; \mathrm{M} 460 \mathrm{I}=4.5 \mathrm{~s}^{-1}$; $\left.\mathrm{H} 520 \mathrm{Q}=8.4 \mathrm{~s}^{-1} ; \mathrm{A} 594 \mathrm{~T}=5.5 \mathrm{~s}^{-1} ; \mathrm{L} 595 \mathrm{~F}=6.4 \mathrm{~s}^{-1}\right)$.

\section{Effects of maribavir on the autophosphorylation of wild type and mutant UL97 species}

In the absence of maribavir, the GCV resistant UL97 mutants H520Q, A594T and L595F exhibited autophosphorylation levels equivalent to the wild type UL97 (Figure 5). However, the M460I mutant consistently exhibited approximately $85 \%$ of the autophosphorylation levels of the wild type UL97 ( $\mathrm{p}=0.01)$. Mutations of the invariant lysine (K355Q) completely negated autophosphorylation, as did mutation of the arginine at amino acid 461. Interestingly, the mutation that confers maribavir resistance to UL97 (L397R) resulted in a reduction in autophosphorylation to approximately $8 \%$ of the wild type levels. The remaining mutations within the UL97 gene (D456A, H662L and V665I) did not result in any change in autophosphorylation compared to that catalysed by the wild type UL97.

In the presence of a fixed concentration of maribavir $(0.5 \mu \mathrm{M})$ the autophosphorylation of every form of UL97 investigated (mutant and wild type) was reduced to below $10 \%$ of the total phosphorylation of the wild type UL97 protein (Table 1). As expected, the autophosphorylation of the L397R mutant, which is resistant to maribavir, was unaffected although the absolute autophosphorylation level of this mutant is already substantially reduced (see above).

Quantitative image analysis of the autoradiographs generated from the protein kinase assays using increasing concentrations of maribavir allowed calculation of the IC50 for each UL97 protein under investigation (Figure 6). The $\mathrm{IC}_{50}$ for wild type UL97 was $34 \mathrm{nM}$, and for the mutants H520Q, A594T, L595F, the D456A, H662L and V665I the IC50 was $33 \mathrm{nM}, 31 \mathrm{nM}, 28 \mathrm{nM} 34 \mathrm{nM}$, $40 \mathrm{nM}$ and $30 \mathrm{nM}$ respectively. There was no autophosphorylation of the $\mathrm{K} 355 \mathrm{Q}$ and N461G mutants hence 


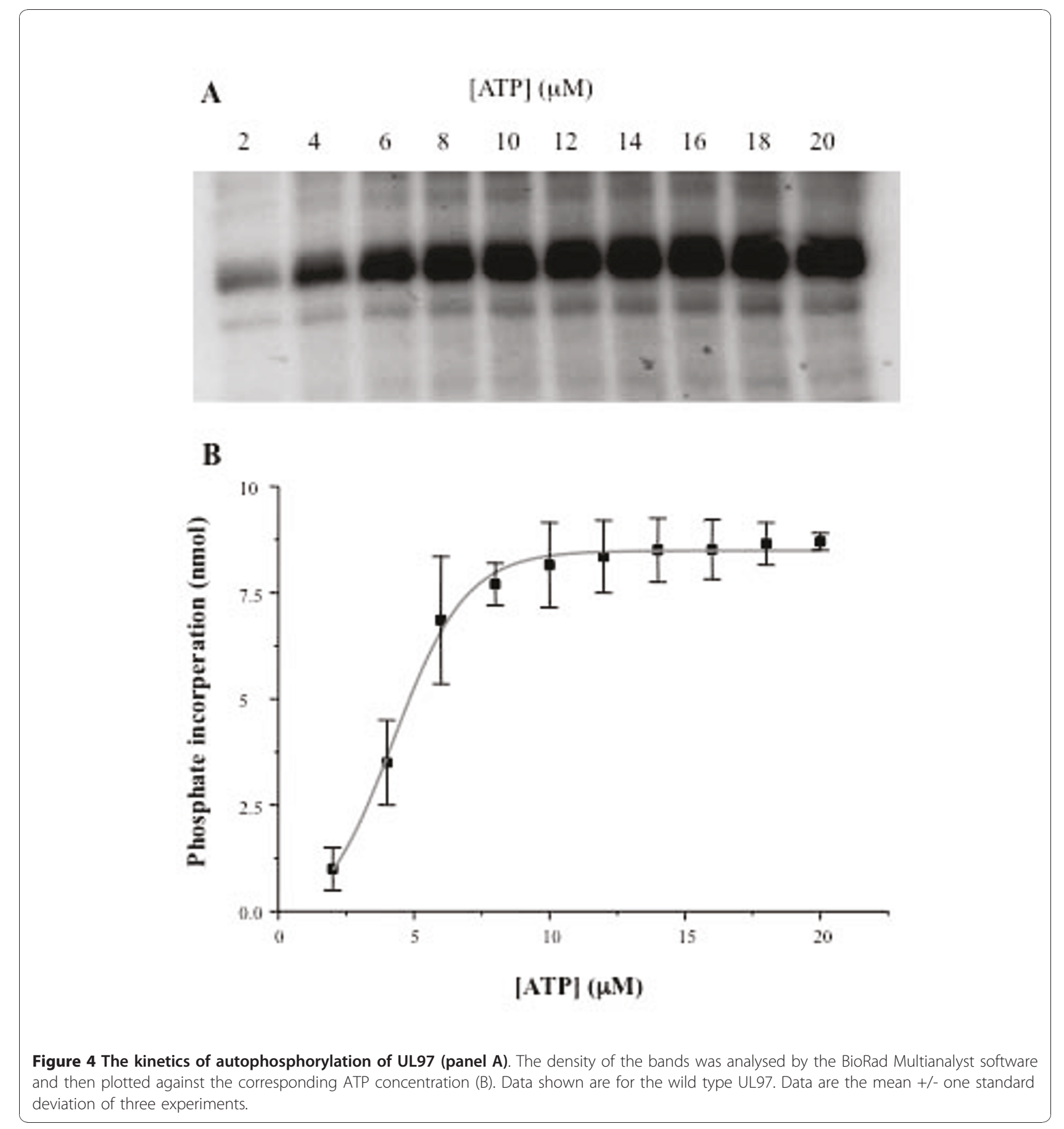

these proteins were not examined in the presence of maribavir. The maribavir-resistant mutant (L397R) did not exhibit a reduction in autophosphorylation even when drug concentrations were increased to $100 \mu \mathrm{M}$. In contrast to the comparable $\mathrm{IC}_{50}$ values observed between wild type UL97 and the GCV resistant mutants at amino acids 520, 594 and 595, the M460I mutant was hypersensitive to maribavir with an IC50 of $4.8 \mathrm{nM}$ (Figure 6).

\section{Mechanism of action of maribavir}

In order to determine whether maribavir was acting as a competitive inhibitor of ATP, a protein kinase assay was performed using the wild type UL97 protein with increasing concentrations of ATP $(2 \mu \mathrm{M}$ to $20 \mu \mathrm{M})$ in the presence of different concentrations of maribavir. The level of UL97 autophosphorylation decreased upon addition of maribavir at each ATP concentration and for each increase in maribavir concentration (Figure 7). 


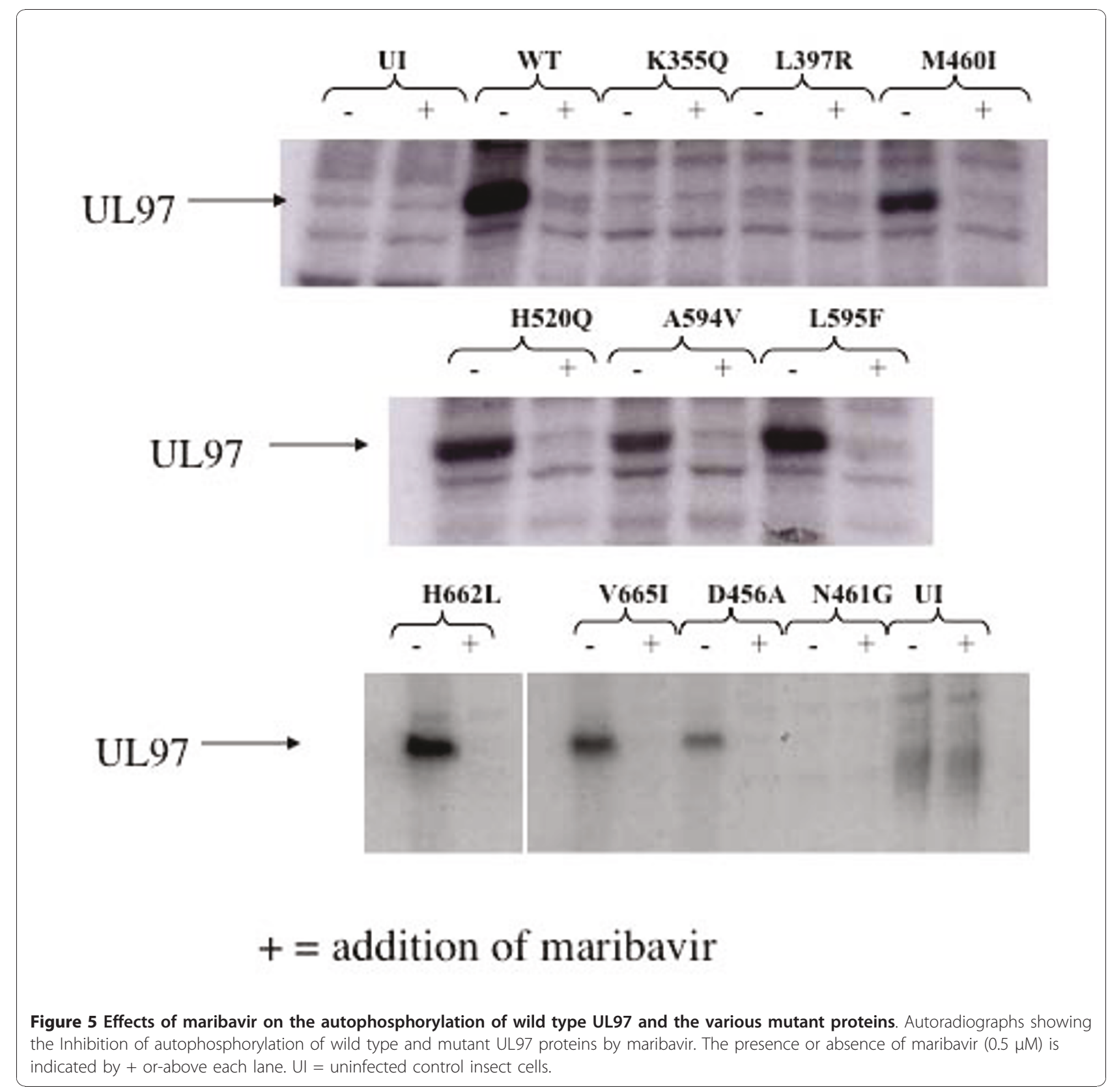

The family of lines observed in the Lineweaver Burke plot shown in Figure 7 crossed the $y$-axis at 1/Vmax and was consistent with maribavir acting as a competitive inhibitor of ATP. The computed Ki for maribavir was $10 \pm 0.8 \mathrm{nM}$.

\section{Discussion}

UL97 is an enigmatic protein which likely possesses a number of roles in the viral life cycle relating to both viral and cellular processes [4-10]. The protein autophosphorylates a number of serine and threonine residues although there is no evidence that it is a substrate for cellular serine/threonine kinses. The availability of a new class of antiviral compounds based upon a benzimidazole core structure that target the kinase activity of UL97 coupled with purified UL97 allowed us to undertake a detailed quantitative biochemical analysis of the functional implications of a spectrum of mutations, including the key mutations giving rise to clinically significant drug resistance against either GCV or maribavir, on both the GCV and protein kinase functions of UL97.

Initially we investigated the function of the mutant UL97 proteins as GCV kinases. Consistent with other studies [46] the results showed that the GCV kinase 
Table 1 Effects of maribavir on the relative levels of autophosphorylation of the wild type and mutant UL97 proteins

\begin{tabular}{lll}
\hline U197 species & \multicolumn{2}{c}{ \% autophosphorylation } \\
\cline { 2 - 3 } & No Maribavir & Plus Maribavir \\
Wild type & 100 & $4.5 \pm 2.0$ \\
K355Q & $1.0 \pm 0.9$ & $0.5 \pm 0.8$ \\
L397R & $7.8 \pm 3.3$ & $7.8 \pm 4.5$ \\
D456A & $104.0 \pm 4.0$ & $5.0 \pm 1.0$ \\
M460V & $86 \pm 2.0$ & $3.9 \pm 2.0$ \\
N461G & $8.0 \pm 1.8$ & $4.2 \pm 2.0$ \\
H520Q & $103.5 \pm 3.5$ & $3.8 \pm 1.5$ \\
A594T & $107.0 \pm 5.0$ & $2.5 \pm 2.5$ \\
L595F & $100.0 \pm 8.0$ & $5.0 \pm 1.0$ \\
H662L & $102.5 \pm 4.5$ & $3.0 \pm 1.5$ \\
V665I & $100.5 \pm 2.0$ & $3.0 \pm 1.5$ \\
\hline
\end{tabular}

The data are normalised relative to wild type and show the mean and standard deviation of triplicate analysis.

efficiency of the well characterised GCV resistant mutants of UL97 (M460I, H520Q and A594V and L595F) was between 10-15\% of the wild type levels. Mutations in other key domains of UL97 showed a variety of GCV kinase activities. The D456A mutant showed similar GCV phosphorylation levels as the wild type protein despite being part of the highly conserved kinase domain whereas the H662L mutant phosphorylated GCV to $82 \%$ of wild type levels. In contrast, the N461G mutant and mutation of the invariant protein kinase lysine at amino acid 355 led to the complete loss of GCV kinase activity while the V665I mutant reduced GCV phosphorylation to $25 \%$ of wild type levels. Interestingly, the maribavir resistant mutant L397R was severely debilitated as a GCV kinase ( $\sim 8 \%$ of wild type levels) implying that this mutant would also be crossresistant to GCV. These data are consistent with recent in vitro studies of this mutant [47].

We next investigated the autophosphorylation of the UL97 proteins. The Km for ATP was similar for both wild type and mutant UL97 proteins $(27 \mu \mathrm{M})$ and was comparable to that observed in previous studies on U69 protein of HHV-6 [48]. However, when the Vmax/Km were compared most of the GCV resistant UL97 proteins appeared to be similar to the wild type in their utilisation of ATP with the exception of the M460I mutation which was less efficient. This observation is consistent with the observation that the autophosphorylation levels observed for the M460I were reduced compared to wild type and other GCV resistant mutants analysed in this study.

Previous data using UL97 mutants of the invariant lysine at codon 355 and the conserved asparagine at codon 461 has shown that UL97 autophosphorylation is a prerequisite for the phosphorylation of GCV [15]. We have extended these analyses to include a substantial range of additional mutants and investigated the effects of maribavir on each mutant species. Interestingly, with the exception of mutations at essential amino acids (L355 and N461) the majority of GCV resistant mutants showed autophosphorylation levels comparable to the wild type. The only exceptions were the M460I mutation which exhibited autophosphorylation at $83 \%$ of wild type levels and the maribavir resistant mutant (L397R) where autophosphorylation was reduced to $\sim 10 \%$ of wild type levels. Thus, certain UL97 mutations can possess both high level protein and GCV kinase activity ((H662L, D456A) or high level protein kinase function and low level GCV kinase activity (M460I, A594T, L595F, H520Q). However, only the L397R mutation exhibited both low protein and low GCV kinase activity. Addition of maribavir resulted in a substantial reduction in the autophosphorylation of wild type and all mutant UL97 proteins except for mutations that either negated protein kinase function (K355Q) or contained the maribavir resistance mutation (L397R). Based upon data for GCV resistant UL97 mutants in vivo [35], the low level of autophosphorylation associated with the L397 mutant would result in this mutant being substantially less fit than wild type virus in vivo. It will be interesting to perform similar autophosphorylation analysis on the recently described additional maribavir resistant mutants $[42,43]$ and extend these studies to determine relative fitness differences between these maribavir resistant viruses and their wild type counterparts.

The $\mathrm{IC}_{50}$ for maribavir of all the UL97 species were very similar with a mean value of $33 \mathrm{nM}(+/-12 \mathrm{nM})$. Importantly, each of the phenotypic GCV-resistant mutants exhibited the equivalent sensitivity to maribavir as the wild type UL97. However, the $\mathrm{IC}_{50}$ of maribavir for the M460I mutant (4.8 nM) showed that this mutant was approximately four times more sensitive to the drug. This observation may reflect the position of the 460 codon in the conserved protein kinase consensus sequence HRDLKXXN (amino acids 454-461 in UL97) that comprises the catalytic loop. Unfortunately, the N461G mutation exhibited a total loss in autophosphorylation and so further investigations were precluded. The fact that the L397R mutation (and the other more recently described mutations [42]) reside in the protein kinase subdomain III, which is involved in the stabilisation of the interaction between the invariant lysine and the $\alpha$ - and $\beta$ - phosphates of the ATP, suggests that maribavir may interfere with this interaction. We showed that the inhibition by maribavir was alleviated at high concentrations of ATP and increasing concentrations of maribavir reduced the incorporation of radiolabelled phosphate in the UL97 autophosphorylation 

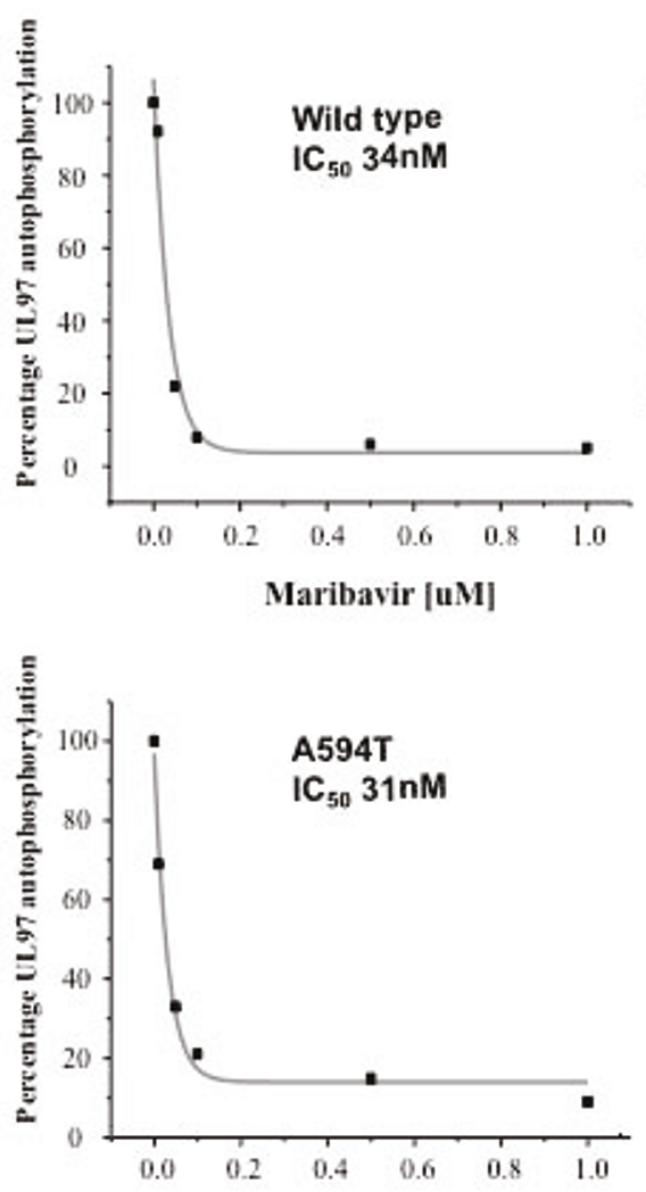

Maribavir [uM]
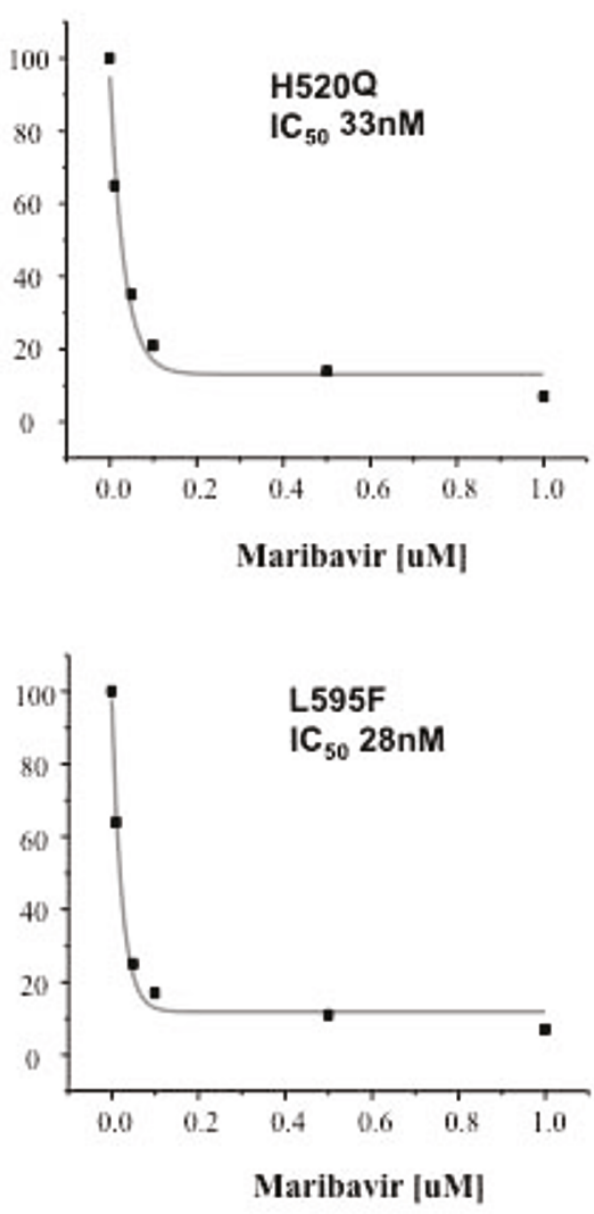

M460

$\mathrm{IC}_{50} 4.8 \mathrm{nM}$

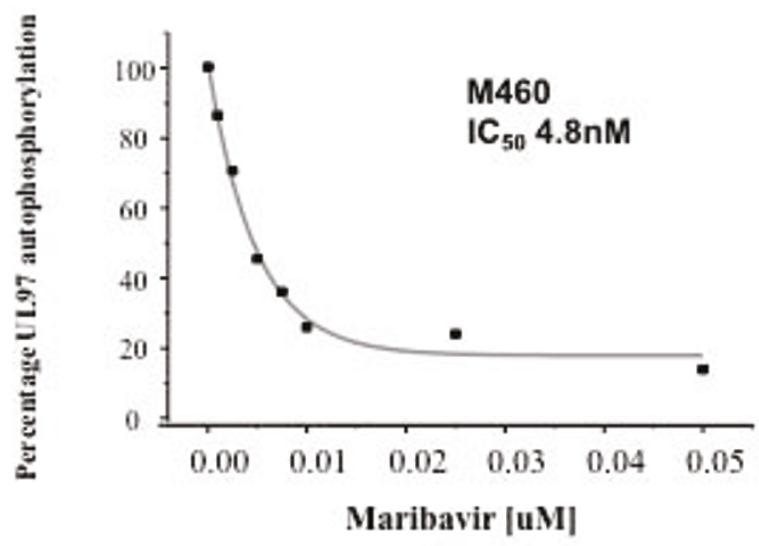

Figure $6 \mathbf{I C}_{50}$ of maribavir for the wild type and mutant UL97 Proteins. The wild type and mutant UL97 proteins were subjected to protein kinase assays with varying concentrations of maribavir (0.01-1.0 $\mathrm{MM}$ ). The autoradiographs were analysed using the BioRad Multianalyst software and UL97 phosphorylation plotted as a percentage of the total phosphorylation in the absence of maribavir. An exponential decay curve was fitted and the $I_{50}$ of maribavir was determined for each of the proteins. The alteration of x-axis scale of the M460I graph should be noted. The $I C_{50}$ for each species is shown. 


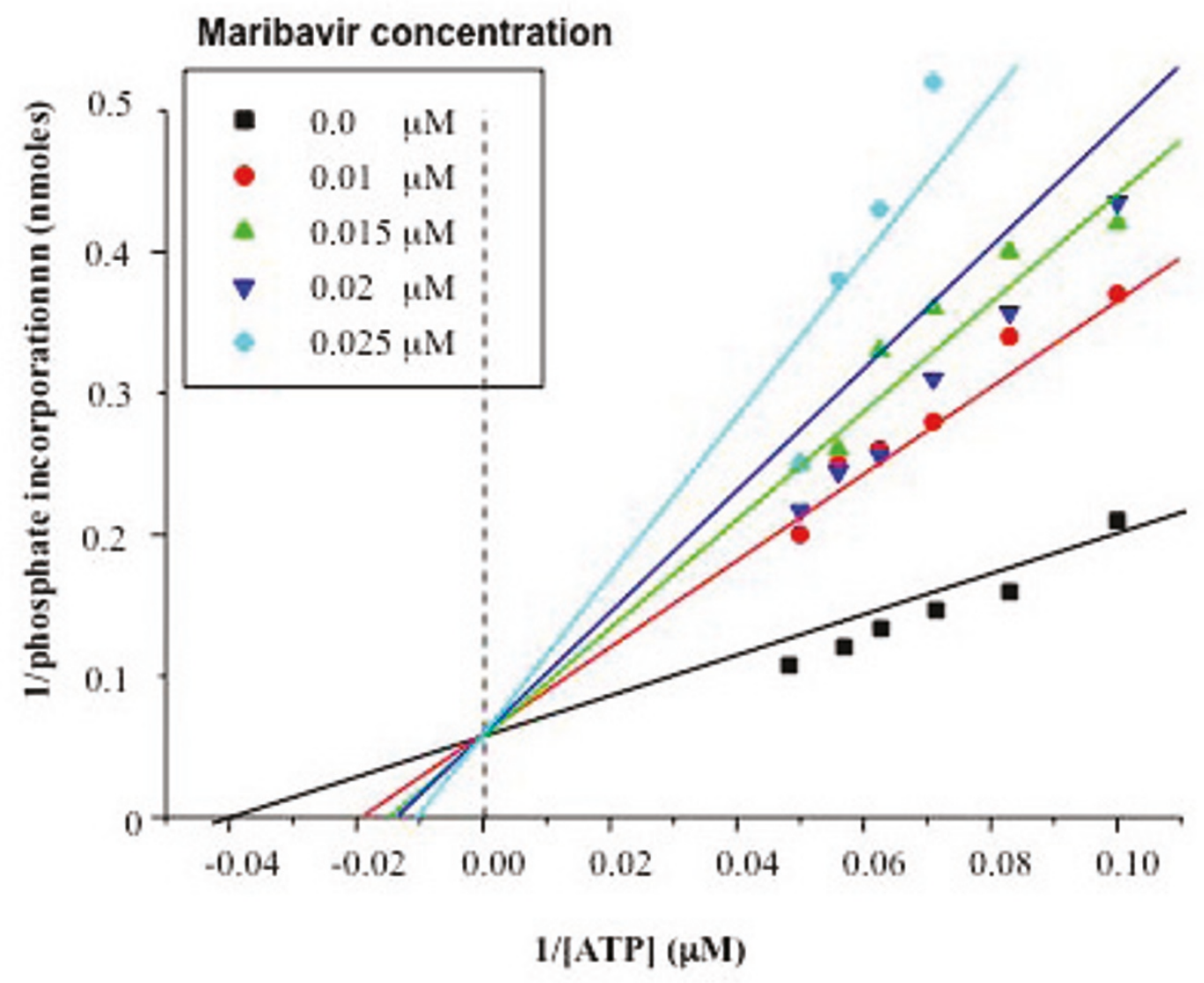

Figure 7 Competitive inhibition of ATP binding by maribavir. Protein kinase assays were performed containing increasing concentrations of ATP $(2 \mu \mathrm{M}-20 \mu \mathrm{M})$ in the absence of maribavir $(0.0 \mu \mathrm{M}$ on the graph) and repeated for increasing maribavir concentrations $(0.01 \mu \mathrm{M}, 0.015 \mu \mathrm{M}$, $0.2 \mu \mathrm{M}$ or $0.25 \mu \mathrm{M})$. The inverse of the velocity of autophosphorylation was plotted against the corresponding inverse of the [ATP] and the Ki value for maribavir determined using standard enzyme kinetic equations for competitive inhibition.

assay. When the Lineweaver-Burke plots were constructed, the resulting family of lines converged on the $\mathrm{Y}$-axis at approximately $1 / \mathrm{V}_{\max }$. These observations were consistent with maribavir acting as a competitive inhibitor of ATP with a computed Ki of $10 \mathrm{nM}$.

In conclusion, we have shown that GCV resistant mutations in the UL97 kinase do not affect the Km of the enzyme for ATP but that the UL97 M460 mutation confers hypersensitivity to maribavir. Maribavir is a potent competetitive inhibitor of ATP and a mutation at L397 that yields maribavir resistance substantially reduces both the GCV kinase and protein kinase activity of UL97 meaning that maribavir resistant strains of HCMV will have to be treated with foscarnet or cidofovir.

\section{Acknowledgements}

We are grateful to Dr Karen Biron (GSK, USA) for provision of maribavir and for useful discussions. We also wish to thank Dr Richard Milne (UCL, London) for constructive comments on this manuscript. This work was supported by grants from the UK Medical Research Council (ROPA award) and through an MRC Centre for Medical Molecular Virology and in part by the Wellcome Trust.

\section{Authors' contributions}

VCE designed the experimental approach, wrote the manuscript and supervised the work. CASL performed the experimental work, helped design the experiments and contributed to the writing of the manuscript. All authors read and approved the final manuscript.

\section{Competing interests}

VCE has acted on advisory boards and as a speaker for Roche Pharmaceuticals. VCE has also acted on advisory boards for Viropharma. No other potential conflicts of interest exist.

Received: 4 August 2010 Accepted: 7 December 2010 Published: 7 December 2010

\section{References}

1. He Z, He YS, Kim Y, Chu L, Ohmstede C, Biron KK, Coen DM: The human cytomegalovirus UL97 protein is a protein kinase that autophosphorylates on serines and threonines. J Virol 1997, 71(1):405-11.

2. van Zeijl M, Fairhurst J, Baum EZ, Sun L, Jones TR: The human cytomegalovirus UL97 protein is phosphorylated and a component of virions. Virology 1997, 231(1):72-80. 
3. Wolf DG, Honigman A, Lazarovits J, Tavor E, Panet A: Characterization of the human cytomegalovirus UL97 gene product as a virion-associated protein kinase. Arch Virol 1998, 143(6):1223-32

4. Prichard MN, Gao N, Jairath S, Mulamba G, Krosky P, Coen DM, Parker BO, Pari GS: A recombinant human cytomegalovirus with a large deletion in UL97 has a severe replication deficiency. J Virol 1999, 73(7):5663-70.

5. Michel D, Schaarschmidt P, Wunderlich K, Heuschmid M, Simoncini L, Mühlberger D, Zimmermann A, Pavić I, Mertens T: Functional regions of the human cytomegalovirus protein pUL97 involved in nuclear localization and phosphorylation of ganciclovir and pUL97 itself. J Gen Virol 1998, 79(Pt 9):2105-12

6. Michel D, Pavić I, Zimmermann A, Haupt E, Wunderlich K, Heuschmid M Mertens T: The UL97 gene product of human cytomegalovirus is an early-late protein with a nuclear localization but is not a nucleoside kinase. J Virol 1996, 70(9):6340-6.

7. Michel D, Kramer S, Höhn S, Schaarschmidt P, Wunderlich K, Mertens T: Amino acids of conserved kinase motifs of cytomegalovirus protein UL97 are essential for autophosphorylation. J Virol 1999, 73(10):8898-901.

8. Krosky PM, Baek MC, Coen DM: The human cytomegalovirus UL97 protein kinase, an antiviral drug target, is required at the stage of nuclear egress. J Virol 2003, 77(2):905-14

9. Hertel L, Chou S, Mocarski ES: Viral and cell cycle-regulated kinases in cytomegalovirus-induced pseudomitosis and replication. PLOS Pathog 2007, 3(1):e6.

10. Azzeh M, Honigman A, Taraboulos A, Rouvinski A, Wolf DG: Structural changes in human cytomegalovirus cytoplasmic assembly sites in the absence of UL97 kinase activity. Virology 2006, 354(1):69-79.

11. Prichard MN, Britt WJ, Daily SL, Hartline CB, Kern ER: Human cytomegalovirus UL97 Kinase is required for the normal intranuclear distribution of pp65 and virion morphogenesis. J Virol 2005, 79(24):15494-502.

12. Prichard MN, Sztul EE, Daily SL, Perry AL, Frederick SL, Gill RB, Hartline CB, Streblow DN, Varnum SM, Smith RD, Kern ER: Human cytomegalovirus UL97 kinase activity is required for the hyperphosphorylation of RB and inhibits the formation of nuclear aggresomes. J Virol 2008, 82:5054-67.

13. Hume AJ, Finkel JS, Kamil JP, Coen DM, Culbertson MR, Kalejta RF: Phosphorylation of retinoblastoma protein by viral protein with cyclindependent kinase function. Science 2008, 320:797-9.

14. Hamirally S, Kamil JP, Ndassa-Colday YM, Lin AJ, Jahng WJ, Baek MC, Noton S, Silva LA, Simpson-Holley M, Knipe DM, Golan DE, Marto JA Coen DM: Viral mimicry of Cdc2/cyclin-dependent kinase 1 mediates disruption of nuclear lamina during human cytomegalovirus nuclear egress. PLOS Pathog 2009, 5(1):e1000275.

15. Baek MC, Krosky PM, Coen DM: Relationship between autophosphorylation and phosphorylation of exogenous substrates by the human cytomegalovirus UL97 protein kinase. J Virol 2002, 76(23):11943-52

16. Krosky PM, Baek MC, Jahng WJ, Barrera I, Harvey RJ, Biron KK, Coen DM, Sethna PB: The human cytomegalovirus UL44 protein is a substrate for the UL97 protein kinase. J Virol 2003, 77(14):7720-7.

17. Kamil JP, Coen DM: Human cytomegalovirus protein kinase UL97 forms a complex with the tegument phosphoprotein pp65. J Virol 2007 81(19):10659-68, Erratum in: J Virol 2007, 81(23):13277.

18. Littler E, Stuart AD, Chee MS: Human cytomegalovirus UL97 open reading frame encodes a protein that phosphorylates the antiviral nucleoside analogue ganciclovir. Nature 1992, 358(6382):160-2.

19. Sullivan V, Talarico CL, Stanat SC, Davis M, Coen DM, Biron KK: A protein kinase homologue controls phosphorylation of ganciclovir in human cytomegalovirus-infected cells. Nature 1992, 358(6382):162-4, Erratum in: Nature 1993 Dec 23-30;366(6457):756. Nature. 1992 Sep 3;359(6390):85.

20. Talarico CL, Burnette TC, Miller WH, Smith SL, Davis MG, Stanat SC, Ng TI, He Z, Coen DM, Roizman B, Biron KK: Acyclovir is phosphorylated by the human cytomegalovirus UL97 protein. Antimicrob Agents Chemother 1999, 43(8):1941-6.

21. Zimmermann A, Michel D, Pavić I, Hampl W, Lüske A, Neyts J, De Clercq E, Mertens T: Phosphorylation of aciclovir, ganciclovir, penciclovir and S2242 by the cytomegalovirus UL97 protein: a quantitative analysis using recombinant vaccinia viruses. Antiviral Res 1997, 36(1):35-42.

22. Paya C, Humar A, Dominguez E, Washburn K, Blumberg E, Alexander B, Freeman R, Heaton N, Pescovitz MD, Valganciclovir Solid Organ Transplant
Study Group: Efficacy and safety of valganciclovir vs. oral ganciclovir for prevention of cytomegalovirus disease in solid organ transplant recipients. Am J Transplant 2004, 4(4):611-20.

23. Asberg A, Humar A, Rollag $H$, Jardine AG, Mouas $H$, Pescovitz MD, Sgarabotto D, Tuncer M, Noronha IL, Hartmann A, VICTOR Study Group: Oral valganciclovir is noninferior to intravenous ganciclovir for the treatment of cytomegalovirus disease in solid organ transplant recipients. Am J Transplant 2007, 7(9):2106-13.

24. Bowen EF, Emery VC, Wilson P, Johnson MA, Davey CC, Sabin CA, Farmer D, Griffiths PD: Cytomegalovirus polymerase chain reaction viraemia in patients receiving ganciclovir maintenance therapy for retinitis. AIDS 1998, 12(6):605-11.

25. Boivin G, Goyette N, Gilbert C, Roberts N, Macey K, Paya C, Pescovitz MD, Humar A, Dominguez E, Washburn K, Blumberg E, Alexander B, Freeman R, Heaton N, Covington E: Absence of cytomegalovirus-resistance mutations after valganciclovir prophylaxis, in a prospective multicenter study of solid-organ transplant recipients. J Infect Dis 2004, 189(9):1615-8.

26. Boivin G, Goyette N, Gilbert C, Humar A, Covington E: Clinical impact of ganciclovir-resistant cytomegalovirus infections in solid organ transplant patients. Transpl Infect Dis 2005, 7(3-4):166-70, Erratum in: Transpl Infect Dis. 2006 Mar;8(1):58

27. Limaye AP, Corey L, Koelle DM, Davis CL, Boeckh M: Emergence of ganciclovir-resistant cytomegalovirus disease among recipients of solidorgan transplants. Lancet 2000, 356(9230):645-9.

28. Chou S, Guentzel S, Michels KR, Miner RC, Drew WL: Frequency of UL97 phosphotransferase mutations related to ganciclovir resistance in clinical cytomegalovirus isolates. J Infect Dis 1995, 172(1):239-42.

29. Chou S, Erice A, Jordan MC, Vercellotti GM, Michels KR, Talarico CL, Stanat SC, Biron KK: Analysis of the UL97 phosphotransferase coding sequence in clinical cytomegalovirus isolates and identification of mutations conferring ganciclovir resistance. J Infect Dis 1995, 171(3):576-83.

30. Baldanti F, Silini E, Sarasini A, Talarico CL, Stanat SC, Biron KK, Furione M, Bono F, Palù G, Gerna G: A three-nucleotide deletion in the UL97 open reading frame is responsible for the ganciclovir resistance of a human cytomegalovirus clinical isolate. J Virol 1995, 69(2):796-800.

31. Chou S, Waldemer RH, Senters AE, Michels KS, Kemble GW, Miner RC, Drew WL: Cytomegalovirus UL97 phosphotransferase mutations that affect susceptibility to ganciclovir. J Infect Dis 2002, 185(2):162-9.

32. Foulongne V, Turriîre C, Diafouka F, Abraham B, Lastere S, Segondy M: Ganciclovir resistance mutations in UL97 and UL54 genes of Human cytomegalovirus isolates resistant to ganciclovir. Acta Virol 2004, 48(1):51-5.

33. Chou S, Marousek G, Parenti DM, Gordon SM, LaVoy AG, Ross JG, Miner RC, Drew WL: Mutation in region III of the DNA polymerase gene conferring foscarnet resistance in cytomegalovirus isolates from 3 subjects receiving prolonged antiviral therapy. J Infect Dis 1998, 178(2):526-30.

34. Baldanti F, Sarasini A, Silini E, Barbi M, Lazzarin A, Biron KK, Gerna G: Four dually resistant human cytomegalovirus strains from AIDS patients: single mutations in UL97 and UL54 open reading frames are responsible for ganciclovir-and foscarnet-specific resistance, respectively. Scand J Infect Dis Supp/ 1995, 99:103-4.

35. Emery VC, Griffiths PD: Prediction of cytomegalovirus load and resistance patterns after antiviral chemotherapy. Proc Natl Acad Sci USA 2000, 97(14):8039-44.

36. Williams SL, Hartline CB, Kushner NL, Harden EA, Bidanset DJ, Drach JC, Townsend LB, Underwood MR, Biron KK, Kern ER: In vitro activities of benzimidazole D-and L-ribonucleosides against herpesviruses. Antimicrob Agents Chemother 2003, 47(7):2186-92

37. Biron KK, Harvey RJ, Chamberlain SC, Good SS, Smith AA, Davis MG, Talarico CL, Miller WH, Ferris R, Dornsife RE, Stanat SC, Drach JC, Townsend LB, Koszalka GW: Potent and selective inhibition of human cytomegalovirus replication by 1263 W94, a benzimidazole L-riboside with a unique mode of action. Antimicrob Agents Chemother 2002, 46(8):2365-72

38. Zacny VL, Gershburg E, Davis MG, Biron KK, Pagano JS: Inhibition of Epstein-Barr virus replication by a benzimidazole L-riboside: novel antiviral mechanism of 5,6-dichloro-2-(isopropylamino)-1-beta-Lribofuranosyl-1H-benzimidazole. J Virol 1999, 73(9):7271-7.

39. Drew WL, Miner RC, Marousek Gl, Chou S: Maribavir sensitivity of cytomegalovirus isolates resistant to ganciclovir, cidofovir or foscarnet. J Clin Virol 2006, 37(2):124-7. 
40. Chou S, Wechel LC, Marousek Gl: Cytomegalovirus UL97 kinase mutations that confer maribavir resistance. J Infect Dis 2007, 196(1):91-4.

41. Chou S, Marousek Gl: Accelerated evolution of maribavir resistance in a cytomegalovirus exonuclease domain II mutant. J Virol 2008, 82(1):246-53.

42. Lu H, Thomas S: Maribavir (ViroPharma). Curr Opin Investig Drugs 2004, 5(8):898-906, Review.

43. Winston DJ, Young JA, Pullarkat V, Papanicolaou GA, Vij R, Vance E, Alangaden GJ, Chemaly RF, Petersen F, Chao N, Klein J, Sprague K, Villano SA, Boeckh M: Maribavir prophylaxis for prevention of cytomegalovirus infection in allogeneic stem-cell transplant recipients: a multicenter, randomized, double-blind, placebo-controlled, dose-ranging study. Blood 2008, 111(11):5403-10

44. Wang LH, Peck RW, Yin Y, Allanson J, Wiggs R, Wire MB: Phase I safety and pharmacokinetic trials of 1263W94, a novel oral anti-human cytomegalovirus agent, in healthy and human immunodeficiency virusinfected subjects. Antimicrob Agents Chemother 2003, 47(4):1334-42.

45. Komazin G, Ptak RG, Emmer BT, Townsend LB, Drach JC: Resistance of human cytomegalovirus to the benzimidazole L-ribonucleoside maribavir maps to UL27. J Virol 2003, 77(21):11499-506.

46. Baldanti F, Michel D, Simoncini L, Heuschmid M, Zimmermann A, Minisini R, Schaarschmidt P, Schmid T, Gerna G, Mertens T: Mutations in the UL97 ORF of ganciclovir-resistant clinical cytomegalovirus isolates differentially affect GCV phosphorylation as determined in a recombinant vaccinia virus system. Antiviral Res 2002, 54(1):59-67.

47. Chou S, Marousek Gl: Maribavir antagonizes the antiviral action of ganciclovir on human cytomegalovirus. Antimicrob Agents Chemother 2006, 50(10):3470-2.

48. Ansari A, Emery VC: The U69 gene of human herpesvirus 6 encodes a protein kinase which can confer ganciclovir sensitivity to baculoviruses. J Virol 1999, 73(4):3284-91.

doi:10.1186/2042-4280-1-4

Cite this article as: Shannon-Lowe and Emery: The effects of maribavir on the autophosphorylation of ganciclovir resistant mutants of the cytomegalovirus UL97 protein. Herpesviridae 2010 1:4.

\section{Submit your next manuscript to BioMed Central} and take full advantage of:

- Convenient online submission

- Thorough peer review

- No space constraints or color figure charges

- Immediate publication on acceptance

- Inclusion in PubMed, CAS, Scopus and Google Scholar

- Research which is freely available for redistribution

Submit your manuscript at www.biomedcentral.com/submit
Ciomed Central 